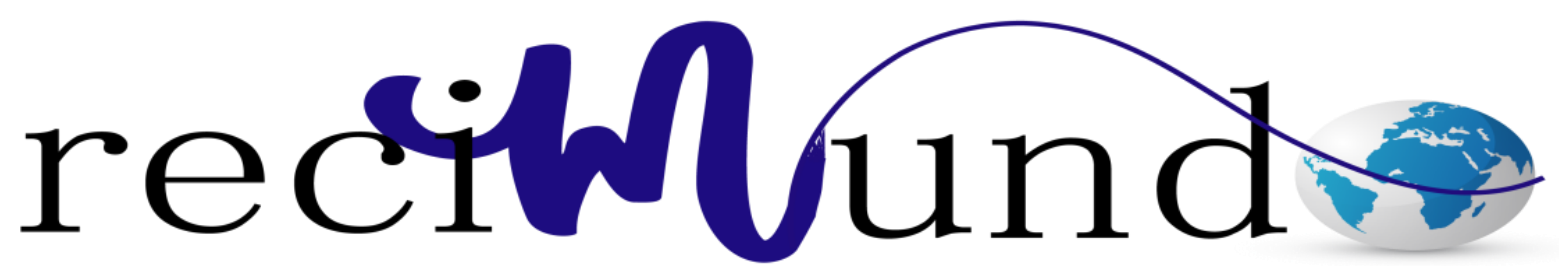

Revista Científica Mundo de la Investigación y el Conocimiento

Gregorio Antonio Moreno Daza ${ }^{\text {a }}$, Linda Beatriz Dávila Solórzano ${ }^{\text {b }}$; Juan José

Moreno Ortega ${ }^{c}$; Franklin Emmanuel Moreno Moreno ${ }^{d}$

Uso de intensificador de imágenes en extracción de cuerpos extraños radiopacos en traumatología

Use of image intensifier in extraction of strange radiopacos bodies in traumatology

Revista Científica Mundo de la Investigación y el Conocimiento. Vol. 2 núm.3, julio, ISSN: 2588-073X, 2018, pp. 500-509

DOI: 10.26820/recimundo/2.(3).julio.2018.500-509

Editorial Saberes del Conocimiento

Recibido: 05/04/2018

Aceptado: 05/06/2018

Publicado: 30/07/2018

Correspondencia: drmorenoneuro@ @otmail.com
a. Medico; drmorenoneuro@hotmail.com
b. Medico; lindbed_2605@hotmail.es
c. Medico; boyjumo@hotmail.com
d. Medico; emmanuel1d1@ hotmail.com 


\section{Uso de intensificador de imágenes en extracción de cuerpos extraños \\ radiopacos en traumatología}

Vol. 2, núm. 3., (2018)

Gregorio Antonio Moreno Daza; Linda Beatriz Davila Solorzano; Juan José Moreno Ortega;

Franklin Emmanuel Moreno Moreno

\section{RESUMEN}

En el presente trabajo de investigación se indagó sobre el uso del intensificador de imágenes en extracción de cuerpos extraños radiopacos en traumatología. Primero que todo hay que aclarar que este método constituye un componente imprescindible y de uso prácticamente generalizado, en el cual se usa radiaciones de bajo nivel de corriente, o sea fluoroscópicas, de manera que puedan aplicarse durante un tiempo considerablemente mayor que el que dura la exposición radiográfica más larga. Esta herramienta funciona con un tubo destinado para este propósito, que es conocido como fluoroscópico. Las radiaciones procedentes del tubo atraviesan al paciente y llegan al seriógrafo, en el que se encuentra montado el intensificador de imágenes o la pantalla fluoroscópica. En el último caso allí termina la cadena, puesto que es sobre dicha pantalla que se forma la imagen y donde directamente el médico observa la región a estudiar. Asimismo, hay que definir que un cuerpo extraño es cualquier elemento ajeno al cuerpo que entra a éste, ya sea a través de la piel o por cualquier orificio natural como los ojos, nariz, garganta, impidiendo su normal funcionamiento. Se pudo obtener como resultado que las ventajas de la navegación por fluoroscopía son la reducción del tiempo quirúrgico y la cantidad de irradiación, que pasa de unos 140 segundos sin navegación a sólo 8 segundos, lo cual supone una diferencia sustancial. Entre las conclusiones se pudo destacar que en el caso de objeto radiopaco es imprescindible disponer de un intensificador de imágenes para localización del cuerpo extraño en el curso de la cirugía, mientras que si se trata de cuerpo extraño radiolúcido es más recomendable la localización a través de la clínica ya que estas tienden a la formación de granulomas.

Palabras claves: Intensificador de imagen, cuerpo extraño, rayos X, cirugía y fluoroscopía. 


\section{Uso de intensificador de imágenes en extracción de cuerpos extraños radiopacos en traumatología}

Vol. 2, núm. 3., (2018)

Gregorio Antonio Moreno Daza; Linda Beatriz Davila Solorzano; Juan José Moreno Ortega; Franklin Emmanuel Moreno Moreno

\section{ABSTRACT}

In the present research work we investigated the use of the image intensifier in the extraction of radiopaque foreign bodies in traumatology. First of all, it is necessary to clarify that this method constitutes an essential component and of practically generalized use, in which radiations of low current level, that is to say fluoroscopic, are used, so that they can be applied for a considerably longer time than the duration of the longer radiographic exposure. This tool works with a tube designed for this purpose, which is known as fluoroscopic. The radiation coming from the tube goes through the patient and reaches the seriograph, where the image intensifier or the fluoroscopic screen is mounted. In the last case there ends the chain, since it is on said screen that the image is formed and where the doctor directly observes the region to be studied. Also, we must define that a foreign body is any element outside the body that enters it, either through the skin or any natural hole such as eyes, nose, throat, preventing normal operation. It was possible to obtain as a result that the advantages of navigation by fluoroscopy are the reduction of surgical time and the amount of irradiation, which goes from about 140 seconds without navigation to only 8 seconds, which is a substantial difference. Among the conclusions it was possible to emphasize that in the case of a radiopaque object, it is essential to have an image intensifier to locate the foreign body in the course of the surgery, whereas if it is a radiolucent foreign body, it is more advisable to locate it through the clinic since these tend to the formation of granulomas.

Key Words: Image intensifier, foreign body, X-rays, surgery and fluoroscopy. 


\section{Uso de intensificador de imágenes en extracción de cuerpos extraños \\ radiopacos en traumatología}

Vol. 2, núm. 3., (2018)

Gregorio Antonio Moreno Daza; Linda Beatriz Davila Solorzano; Juan José Moreno Ortega;

Franklin Emmanuel Moreno Moreno

\section{Introducción.}

Los sistemas intensificadores electrónicos de imágenes empleados en radiología, constituyen un componente imprescindible y de uso prácticamente generalizado. En este caso se usan radiaciones de bajo nivel de corriente, o sea fluoroscópicas, de manera que puedan aplicarse durante un tiempo considerablemente mayor que el que dura la exposición radiográfica más larga.

El tubo destinado para este propósito es el conocido como fluoroscópico, el cual se ubica bajo la mesa en los sistemas convencionales. Las radiaciones procedentes del tubo atraviesan al paciente y llegan al seriógrafo, en el que se encuentra montado el intensificador de imágenes o la pantalla fluoroscópica. En el último caso allí termina la cadena, puesto que es sobre dicha pantalla que se forma la imagen y donde directamente el médico observa la región a estudiar. (Romo, 2010)

De este modo se desarrollaron los sistemas de intensificación de imagen, en los que partiendo de la idea original de la pantalla fluoroscópica, se consiguió mejorar la imagen obtenida. Gracias a este sistema se elimina la necesidad de adaptación del observador a la oscuridad y, en definitiva, permite realizar exploraciones más fiables y con un mejor rendimiento de la radiación. (Camacho, 2003)

Las pantallas de refuerzo capturan los fotones de Rx y los convierten en fotones de luz visible, trasmiten esa luz a la película, aprovechando una de las propiedades de los Rx, que es la de producir fluorescencia en ciertas sustancias. La fluorescencia es la capacidad que tienen 


\section{Uso de intensificador de imágenes en extracción de cuerpos extraños radiopacos en traumatología}

Vol. 2, núm. 3., (2018)

Gregorio Antonio Moreno Daza; Linda Beatriz Davila Solorzano; Juan José Moreno Ortega; Franklin Emmanuel Moreno Moreno

ciertos compuestos llamados fósforos, de emitir instantáneamente luz, cuando inciden sobre ellos

Rx. De esta manera se consigue irradiar menos al paciente disminuyendo el tiempo de exposición, en definitiva se ha conseguido disminuir los valores de exposición, optimizando la dosis que recibe el paciente. (Martínez, 2006)

La fluoroscopia permite ubicar la lesión o zona del cuerpo donde se tiene que realizar un procedimiento médico, como colocar una inyección o hacer una cirugía. Al observar específicamente el lugar del cuerpo se tiene mayor exactitud y los resultados de los métodos médicos son más efectivos.

Asimismo, un cuerpo extraño es cualquier elemento ajeno al cuerpo que entra a éste, ya sea a través de la piel o por cualquier orificio natural como los ojos, nariz, garganta, impidiendo su normal funcionamiento. (Xunta de Galicia , 2016).

Unas de las posibilidades de las heridas articulares es la penetración de un cuerpo extraño en la articulación, no fácil de detectar si no se hace una exploración profunda de la herida y un estudio radiográfico. Las consecuencias de un cuerpo extraño articular van más allá de una lesión mecánica de las superficies articulares, pude ser causa de una grave artritis por contaminación del recinto articular. (Rodriguez, Zazo, Pareja, Herreros, \& Garcia, 2005)

\section{Materiales y métodos.}

Para llevar adelante el siguiente trabajo académico se recurrió al método de la investigación documental. Esta herramienta se fundamenta en la recolección de documentos, 


\section{Uso de intensificador de imágenes en extracción de cuerpos extraños \\ radiopacos en traumatología}

Vol. 2, núm. 3., (2018)

Gregorio Antonio Moreno Daza; Linda Beatriz Davila Solorzano; Juan José Moreno Ortega;

Franklin Emmanuel Moreno Moreno

categorizarlos, analizarlos; para después poder presentar un resultado coherente. El objetivo de esta metodología es poder aportar nuevos conocimientos. (Sampieri, 1998)

También se puede decir que esta estrategia se basa en la observación y reflexión sistemática de realidades teóricas y empíricas, en la que usa diversos tipos de documentos que sirven para indagar, interpretar, exponer cifras e informaciones referentes al tema de ciencia que se trabaje. Todo utilizando instrumentos que tienen como meta conseguir resultados que pueden ser la plataforma para otra exploración científica y la construcción de nuevos conocimientos. (Martínez S. , 2002)

De igual forma, se puede enumerar en los siguientes pasos: realizar un proceso de abstracción científica, generalizando sobre la base de lo fundamental; utilizar los procedimientos lógicos y mentales de toda investigación; tales como: análisis, síntesis, deducción, inducción, entre otros; y se debe a una recopilación adecuada de datos, que permiten redescubrir hechos, sugerir problemas, ubicar hacia otras fuentes de investigación, orientar formas para elaborar instrumentos de investigación y elaborar hipótesis (Rodríguez, 2013)

Para llevar adelante este estudio se utilizó la metodología de la investigación documental para recopilar la información necesaria para obtener resultados, sacar conclusiones y poder brindar recomendaciones. Esto es imprescindible, ya que investigar implica conocer los antecedentes y el estado del arte del objeto de estudio. Los antecedentes están referidos a las investigaciones ya realizadas acerca del tema, y cuyos resultados deben tener en cuenta. (Parraguez, Chunga, Flores, \& Romero, 2017) 


\section{Uso de intensificador de imágenes en extracción de cuerpos extraños radiopacos en traumatología}

Vol. 2, núm. 3., (2018)

Gregorio Antonio Moreno Daza; Linda Beatriz Davila Solorzano; Juan José Moreno Ortega; Franklin Emmanuel Moreno Moreno

Dicho estudio se respaldó con una investigación bibliográfica permite, entre otras cosas, evitar iniciar exploraciones anteriormente realizadas, obtener conocimiento de experimentos previos para repetirlos en caso de ser necesario, buscar datos sugerentes, culminar investigaciones interrumpidas o incompletas, seleccionar los materiales y documentos para un marco teórico. (Rodríguez, 2013)

Con lo expuestos se puede asegurar que en el presente trabajo de investigación se utilizó como metodología una revisión bibliográfica documental no experimental, puesto que realizó una revisión y análisis de diferentes artículos médicos y de opinión referentes a la a prevalencia las convulsiones febriles, para poder abordar de la mejor manera el uso del intensificador de imágenes en extracción de cuerpos extraños radiopacos en traumatología.

\section{Resultados.}

Las ventajas de la navegación por fluoroscopía son la reducción del tiempo quirúrgico y la cantidad de irradiación, que pasa de unos 140 segundos sin navegación a sólo 8 segundos, lo cual supone una diferencia sustancial. Con respecto a la duración de la intervención, es entre un 10 y un 15 por ciento más rápida, lo cual es clave, teniendo en cuenta que alargar el tiempo quirúrgico aumenta el riesgo de infección. La fuente de dolor es identificada y localizada con precisión. La técnica puede ser empleada a través de todo el cuerpo, incluso pacientes con dificultades anatómicas pueden ser inyectados con un mínimo de riesgo. Además, pueden utilizarse agujas delgadas imponiendo menor riesgo y mejorando el confort del paciente. (Prieto, 2010) 


\section{Uso de intensificador de imágenes en extracción de cuerpos extraños \\ radiopacos en traumatología}

Vol. 2, núm. 3., (2018)

Gregorio Antonio Moreno Daza; Linda Beatriz Davila Solorzano; Juan José Moreno Ortega;

Franklin Emmanuel Moreno Moreno

De igual manera, si bien ésta es una técnica que tiene muchos beneficios no se puede obviar el hecho que se trabaja con radiación como ya se ha desarrollado anteriormente y que la misma tiene efectos dañinos para la salud del paciente cuando es utilizada a altas dosis o de forma repetitiva, la misma se encuentra relacionada con patologías como cáncer, malformaciones fetales, entre otras. Hay que tomar en cuenta precauciones previo a la realización del procedimiento y al momento de ejecutar el mismo. Es importante destacar igualmente que dicho procedimiento sólo debe ser realizado en manos de profesionales en el área con experiencia comprobada, para así evitar efectos adversos y complicaciones. Conclusiones

En el caso de objeto radiopaco es imprescindible disponer de un intensificador de imágenes para localización del cuerpo extraño en el curso de la cirugía, mientras que si se trata de cuerpo extraño radiolúcido es más recomendable la localización a través de la clínica ya que estas tienden a la formación de granulomas. (Sánchez, 1992)

Cada protocolo clínico debe incluir el control de la dosis administrada tanto para el paciente como para el Trabajador Ocupacionalmente Expuesto (TOE), la estimación de la dosis absorbida máxima recibida por el paciente, descripción del tipo de intervención, registro de las dosis acumulativas, entre otros. Los intervencionistas deben estar capacitados para utilizar la información de la dosis en piel y las técnicas para controlar la dosis absorbida que puedan excederse de 100 cGy (1Gy), tomando en cuenta que posiblemente el estudio puede eventualmente repetirse, por lo que es recomendable que cualquier procedimiento sea asentado en la historia clínica y así poder optimizar la práctica haciendo un seguimiento adecuado de estos casos. (Rivas, 2017) 


\section{Uso de intensificador de imágenes en extracción de cuerpos extraños radiopacos en traumatología}

Vol. 2, núm. 3., (2018)

Gregorio Antonio Moreno Daza; Linda Beatriz Davila Solorzano; Juan José Moreno Ortega; Franklin Emmanuel Moreno Moreno

\section{Recomendaciones}

El riesgo de infección y diferentes complicaciones aumenta a medida que el cuerpo extraño permanece más tiempo dentro del cuerpo ya que puede arrastrar fragmentos de ropa, piel y pelo al interior. Radicando en esto la importancia de la resolución temprana y extracción del cuerpo extraño.

La dosis de radiación que recibe el paciente es alta, cuando se le realiza el estudio de fluoroscopia. Por lo que se limita el uso de esta técnica médica solo para casos específicos y necesarios.

\section{Bibliografía}

Camacho, D. (2003). FLUOROSCOPIA EN QUIRÓFANO. San Martin , Argentina.

Martínez, A. (2006). Radiología: De la imagen convencional a la digital . San Martin , Argentina.

Martínez, S. (2002). Guía de apuntes básicos para el docente de la materia técnicas de investigación. Oaxaca: Grupo emergente de investigación Oaxaca.

Parraguez, S., Chunga, G., Flores, M., \& Romero, R. (2017). El estudio y la invstigacion documental: estrategias metodologicas y herramientas tic . Chiclayo: EMDOCOSEGE S.A.

Prieto, A. (2010). Fluoroscopía. Cenetec, 5-7.

Rivas, V. (2017). Comparación de la Dosis Absorbida en Piel de Pacientes Sometidos a Estudios de Hemodinamia en Equipos con Intensificador de Imágenes y Flat Panel con películas radiocrómicas . Saber ucv, 25-40. 


\section{Uso de intensificador de imágenes en extracción de cuerpos extraños \\ radiopacos en traumatología}

Vol. 2, núm. 3., (2018)

Gregorio Antonio Moreno Daza; Linda Beatriz Davila Solorzano; Juan José Moreno Ortega;

Franklin Emmanuel Moreno Moreno

Rodriguez, C., Zazo, M., Pareja, L., Herreros, R., \& Garcia, M. (2005). Lesión por cuerpo extraño en rodilla. REVISTA ESPAÑOLA DE CIRUGÍA OSTEOARTICULAR, 2-4.

Rodríguez, S. (2013). Acerca de la investigación bibliógrafica y documental. Obtenido de Guia de Tesis: http://guiadetesis. wordpress. com/2013/08/19/acerca-de-la-investigacionbibliografica-ydocumental

Romo, R. (2010). Obtenido de Departamento de Electrónica, Automática y Bioingeniería.: http://dea.unsj.edu.ar/imagenes/recursos/Rx-Intensificadores.PDF

Sampieri, R. (1998). Metodologia de la investigacion. México DF: Interamericana editores.

Sánchez, J. (1992). Manual de Especialidades quirúrgicas . Medical .

Xunta de Galicia . (2016). Primeros Auxilios. Obtenido de Cuerpos extraños: http://www.edu.xunta.gal/centros/iespintorcolmeiro/system/files/5.CUERPOS+EXTRA $\%$ C3\%91OS.pdf 